Alexandre Lima Jukka Takala

\section{Clinical significance of monitoring perfusion in non-vital organs}

Received: 14 May 2014

Accepted: 15 May 2014

Published online: 29 May 2014

(C) Springer-Verlag Berlin Heidelberg and ESICM 2014

\section{A. Lima (®)}

Department of Intensive Care Adults, Erasmus MC University Medical Centre, P.O. Box 2040, Room H625, 3000 CA Rotterdam, The Netherlands

e-mail: a.pintolima@erasmusmc.nl

Tel.: +31638211985

\section{J. Takala}

Department of Intensive Care Medicine, University Hospital Bern (Inselspital), 3010 Bern, Switzerland

e-mail: jukka.takala@insel.ch

Tel.: +41-31-632 4144

Monitoring circulation should help to reveal the risk of hypoperfusion of vital organs, and to guide treatment to restore and maintain hemodynamic stability. The wide spectrum of diagnostic and monitoring technologies has helped intensivists to better understand the complex pathophysiology of acute circulatory failure. A common belief is that hypoperfusion may persist despite apparent stabilization of macrovascular hemodynamics. Various approaches have been introduced to avoid such hypoperfusion-mostly with disappointing or controversial results in the ICU patients. These include supranormal oxygen delivery, treatments based on gastrointestinal tonometry, mixed or central venous saturation, systemic hemodynamics and derived values of cardiac and intrathoracic volumes.

Recently, monitoring other vascular beds susceptible to hypoperfusion has received increasing attention. It is somewhat ironic that first the development of techniques enabling continuous monitoring of peripheral perfusion shifted our focus back to perfusion in non-vital organs, such as the skin, as a marker of perfusion deficits. An observation by British surgeon Jordan Furneaus early in the eighteenth century who wrote one of the first elaborate descriptions of abnormalities in peripheral perfusion during shock conditions [1]. In his description, he emphasized the cold, clammy and mottled skin associated with high heart rate observed in war casualties. Today, monitoring of the peripheral circulation of the skin, subcutaneous tissue and muscle can be done noninvasively in contrast to the need for intravascular catheters, transoesophageal probe insertion, blood component analysis or penetration of the skin used in more traditional hemodynamic monitoring in the ICU [2]. Paradoxically, the technical advancements in peripheral tissue perfusion monitoring were necessary for the renaissance of the most basic approach to peripheral tissue perfusion assessment, clinical examination, in the scientific literature. From all these noninvasive techniques, physical examination of peripheral perfusion based on touching the skin or measuring capillary refill time (CRT) has been best related to the prognosis of patients with circulatory shock. Over the past 30 years, the definition of a delayed CRT has been debated in the literature [3,4]. Although the most useful threshold of CRT in critically ill patients is still a matter of debate, sustained prolongation of CRT after initial hemodynamic optimization in the intensive care unit (ICU) helped to discriminate hemodynamically stable patients with more severe organ dysfunction, other than circulatory [5]. In addition, patients with a prolonged CRT had significantly higher odds of worsening organ failure than patients with normal CRT. These observations were made in a mixed ICU patient population, and their applicability in specific types of shock, such as septic shock, has not been addressed so far.

The recent paper by Ait-Oufella [6] investigated the skin CRT in a selected group of septic shock patients. Skin CRT of a finger and knee was a strong predictor of 
Table 1 Interobserver agreement between three observers for CRT measurement in three consecutive days in 111 post-operative patients

\begin{tabular}{llll}
\hline & Day 1 & Day 2 & Day 3 \\
\hline Observers: 1 vs. 2 & 0.80 & 0.77 & 0.79 \\
& {$[0.62 ; 0.97]$} & {$[0.53 ; 1.0]$} & {$[0.51 ; 1.0]$} \\
Observers: 1 vs. 3 & 0.85 & 0.89 & 0.70 \\
& {$[0.75 ; 0.95]$} & {$[0.57 ; 0.95]$} & {$[0.45 ; 0.93]$} \\
Observers: 2 vs. 3 & 0.95 & 0.77 & 0.70 \\
& {$[0.85 ; 1.0]$} & {$[0.53 ; 1.0]$} & {$[0.45 ; 0.93]$} \\
\hline
\end{tabular}

14-day mortality. CRT was evaluated in 59 patients at intensive care admission and after initiation of vasopressor therapy within $24 \mathrm{~h}$. Two intensivists blinded to patient treatment quantified the CRT, and the inter-rater concordance was $80 \%$ (73-86) for finger CRT and $95 \%$ (93-98) for knee CRT. Patients who persisted with CRT $>5 \mathrm{~s}$ had an OR of dying in 14 days of $9(95 \%$ CI $1-61)$ when measured in the finger and OR of $23(95 \%$ CI 11-1,568) when measured in the knee. Among patients who died, both knee and index CRT did not change and remained prolonged in $82 \%$ of patients despite resuscitation, demonstrating that abnormal signs of peripheral perfusion in patients with no other clinical signs of shock are predictive of progressing organ dysfunction. These findings have at least two relevant clinical implications. First, CRT is not a subjective but rather a highly reproducible objective measurement, when performed in a standardized way-otherwise its clinical value would be limited. Second, if the clinician stops the resuscitation after the traditional end points of systemic hemodynamics have been normalized, the majority of patients might still remain in a state of persistent hypoperfusion, with the associated increased risk of death. The Ait-Oufella's results extend the recent findings of a single-centre study by van Genderen et al., which showed substantial inter-rater agreement of CRT measurement in 111 post-operative patients for major abdominal surgery (Table 1) during three consecutive days in the ICU [7]. With a substantial inter-rater agreement of CRT measurement, these complementary studies provide convincing evidence of CRT reproducibility, and support the strategy of bedside evaluation of peripheral perfusion with CRT.

Ait-Oufella's results also align with two previous findings by the same group. To analyse mottling objectively, Ait-Oufella et al. [8] recently developed a clinical scoring system based on the area of mottling from the knees to the periphery, and found that a higher mottling score within $6 \mathrm{~h}$ after initial resuscitation was a strong predictor of 14-day mortality during septic shock. In a second investigation, applying the same mottling score system, the same group found that tissue oxygen saturation $(\mathrm{StO} 2)$ measured around the knee in septic shock was associated with an increase of the mottling score [9]. Mottling is the result of heterogenic small vessel vasoconstriction and is thought to reflect abnormal skin perfusion and microcirculatory abnormalities. In this regard, cutaneous vasomotion is one determinant of StO2derived measurements of tissue oxygenation in critically ill patients.

With so much emphasis being given to the global variables of perfusion and the relevance of vital organ perfusion, should we now shift our attention to less vital organs, such skin or muscle? Clearly, vital organ perfusion remains vital. Nevertheless, it is obvious that the circulation of peripheral vascular beds is among the first to deteriorate and the last to be restored after resuscitation in common scenarios of shock. The evaluation of peripheral perfusion is feasible and reproducible, and can be easily used by the whole ICU team involved in the care of patients with acute circulatory problems. Reliance on simple methods, such as CRT, skin temperature and mottling score, must be emphasized and exploited. The true clinical implications of this strategy may be better defined in clinical trials of peripheral perfusion targeted resuscitation. One next logical step, therefore, would be incorporating therapeutic strategies into resuscitation protocols that aim at normalizing the peripheral perfusion parameters to investigate the impact of peripheral perfusion targeted resuscitation on survival of critically ill patients.

Conflicts of interest On behalf of all authors, the corresponding author states that there is no conflict of interest.

\section{References}

1. Jordan F (1867) On shock after surgical operations and injuries. Br Med J 1(319):136-137

2. Lima A, Bakker J (2005) Noninvasive monitoring of peripheral perfusion. Intensive Care Med 31(10):1316-1326

3. Champion HR, Sacco WJ, Copes WS, Gann DS, Gennarelli TA, Flanagan ME (1989) A revision of the trauma score. J Trauma 29(5):623-629
4. Schriger DL, Baraff L (1988) Defining normal capillary refill: variation with age, sex, and temperature. Ann Emerg Med 17(9):932-935

5. Lima A, Jansen TC, van Bommel J, Ince C, Bakker J (2009) The prognostic value of the subjective assessment of peripheral perfusion in critically ill patients. Crit Care Med 37(3):934-938
6. Ait-Oufella H (2014) Capillary refill time exploration during septic shock. Intensive Care Med. doi:10.1007/ s00134-014-3326-4 
7. van Genderen M, Paauwe J, de Jonge J, 8. Ait-Oufella H, Lemoinne S, Boelle PY, van derValk RJP, Lima A, Bakker J et al (2014) Clinical assessment of the peripheral perfusion to early predict postoperative complications after major abdominal surgery: a prospective observational study in adults. J Crit Care (in press) Galbois A, Baudel JL, Lemant J et al 37(5):801-807 in septic shock. Intensive Care Med
9. Ait-Oufella H, Joffre J, Boelle PY, Galbois A, Bourcier S, Baudel JL et al (2012) Knee area tissue oxygen saturation is predictive of 14-day mortality in septic shock. Intensive Care Med 38(6):976-983 